NBER WORKING PAPER SERIES

\title{
TRANSPARENCY OF INFORMATION AND COORDINATION IN ECONOMIES WITH INVESTMENT COMPLEMENTARITIES
}

\author{
George-Marios Angeletos \\ Alessandro Pavan
}

Working Paper 10391

http://www.nber.org/papers/w10391

\author{
NATIONAL BUREAU OF ECONOMIC RESEARCH \\ 1050 Massachusetts Avenue \\ Cambridge, MA 02138 \\ March 2004
}

This paper was prepared for the annual meeting of the American Economic Association and will appear in American Economic Review 94:1 (Papers and Proceedings). We are grateful to Narayana Kocherlakota for the invitation. For useful comments, we thank Daron Acemoglu, Ricardo Caballero, Christian Hellwig, Bengt Holmstrom and Ivan Werning. The views expressed herein are those of the author and not necessarily those of the National Bureau of Economic Research.

(C2004 by George-Marios Angeletos and Alessandro Pavan. All rights reserved. Short sections of text, not to exceed two paragraphs, may be quoted without explicit permission provided that full credit, including (C) notice, is given to the source. 
Transparency of Information and Coordination in Economies with Investment George-Marios Angeletos and Alessandro Pavan

NBER Working Paper No. 10391

March 2004

JEL No. D6, D8, E6

\section{ABSTRACT}

How do public and private information affect equilibrium allocations and social welfare in economies with investment complementarities? And what is the optimal transparency in the information conveyed, for example, by economic statistics, policy announcements, or news in the media? We first consider an environment where the complementarities are weak so that the equilibrium is unique no matter the structure of information. An increase in the precision of public information may have the perverse effect of increasing aggregate volatility. Nevertheless, as long as there is no value to lotteries, welfare unambiguously increases with an increase in either the relative or the absolute precision of public information. Hence, full transparency is optimal. This is because more transparency facilitates more effective coordination, which is valuable from a social perspective. On the other hand, when complementarities are strong enough that multiple equilibria are possible, more transparency permits the market to coordinate more effectively on either the bad or the good equilibrium. In this case, constructive ambiguity becomes optimal if there is a high risk that more transparency will lead to coordination failures.

George-Marios Angeletos

Department of Economics

MIT

50 Memorial Drive, E51-251

Cambridge, MA 02142

and NBER

angelet@mit.edu

Alessandro Pavan

alepavan@northwestern.edu 


\section{Introduction}

Economies with production externalities, demand spillovers, incomplete financial markets, and Keynesian frictions are only a few examples where macroeconomic complementarities play a prominent role. Within this class of economies, how does the precision of publicly provided and privately collected information affect equilibrium allocations and social welfare? And what is the optimal transparency in the information conveyed, for example, by economic statistics, policy announcements, or news in the media? To answer these questions, we consider a simple real economy where the individual return to investment is increasing in the aggregate level of investment and where market participants have heterogenous expectations about the underlying economic fundamentals (the exogenous productivity). We interpret an increase in the transparency of public information either as a reduction in the level of common uncertainty for given level of idiosyncratic uncertainty (that is, an increase in the absolute precision of public information), or as a reduction in the heterogeneity of expectations across market participants for given level of overall uncertainty (that is, an increase in the relative precision of public information).

We first consider an environment where complementarities are weak so that the equilibrium is unique no matter the structure of information. Like in Morris and Shin (2002), complementarities increase the sensitivity of equilibrium allocations with respect to public information, which increases the volatility generated by common noise in market expectations. Moreover, when information is heterogeneous, an increase in the precision of public information may have the perverse effect of increasing aggregate volatility, by increasing the sensitivity of economic activity to common noise. On the contrary, an increase in the precision of private information necessarily reduces aggregate volatility. Nevertheless, we show that, as long as there is no value to lotteries, welfare unambiguously increases with an increase in either the relative or the absolute precision of public information. Hence, policies that either disseminate more precise information about economic fundamentals, or reduce the heterogeneous interpretation of economic statistics and policy measures, necessarily boost welfare. On the contrary, an increase in the precision of private information may reduce welfare by increasing the heterogeneity of expectations and thereby obstructing coordination in the market, in which case policies that discourage the private collection of private information may increase welfare.

Morris and Shin (2002) have recently argued that, in environments with strategic complementarities and heterogeneous information, more precise public information can reduce social welfare, whereas more precise private information is always beneficial. We find rather the opposite. The 
difference in the results is due to an important distinction between the environments in the two models. Morris and Shin (2002) consider a kind of "beauty contest," where the payoff of a player decreases with the distance between his own action and the action of others, but where this distance is irrelevant from a social perspective. It follows that the complementarity is present only at the private level and hence the attempt of the agents to align their actions is socially wasteful. In this case, more transparent public information facilitates more effective coordination, which is valued by the market but not by the society. In contrast, we consider environments where the complementarity is present at the social level so that effective market coordination is socially valuable, as it is likely to be the case in economies with production and demand spillovers, network externalities, or incomplete financial markets. As shown in Angeletos and Pavan (2003), market participants use public information to align their investment choices, but not enough as compared to what is socially optimal, for they do not internalize the positive externality of their investment on the return to others. As a consequence, more transparent public information, by permitting more effective coordination in the market, necessarily increases welfare, despite the fact that it may lead to higher volatility. ${ }^{1}$

In the light of these results, we finally consider the possibility that complementarities are strong enough that multiple equilibria emerge for certain structures of information, in which case more effective coordination in the market need not always be socially beneficial. Indeed, there is a critical threshold for the transparency of public information above which multiple equilibrium levels of investment are possible. Above this threshold, the desirability of more effective market coordination and thus the welfare effect of more transparent public information depend critically on which equilibrium is selected. If the market coordinates on the socially desirable equilibrium, facilitating coordination is beneficial, and welfare tends to be maximized at high levels of transparency. If instead the market coordinates on the undesirable equilibrium, impeding coordination by introducing noise in public information can be welfare enhancing.

This final result is related to Angeletos, Hellwig and Pavan (2003). They show that, in coordination environments where a privately-informed policy maker is interested in fashioning market outcomes, active policy intervention may lead to policy traps, where the optimal policy and market outcomes are dictated largely by arbitrary self-fulfilling market expectations. In the present paper,

\footnotetext{
${ }^{1}$ In independent parallel work, Hellwig (2003) and Lorenzoni (2003), building on Woodford (2002), examine monetary economies in which complementarities arise in pricing decisions. They also find that the Morris-Shin result about the social value of public information can be reversed. However, they do not show how the welfare effects of public information depend on whether market coordination is socially desirable.
} 
we do not consider active policy intervention. Nevertheless, a similar trap emerges regarding the information disseminated by government agencies and central bankers: The optimal transparency depends on the aggressiveness or leniency of market expectations.

We conclude that, in the class of environments considered in this paper, ${ }^{2}$ noise in public information may be socially desirable only when there is a high risk that more transparency will introduce coordination failures. Otherwise, the timely and frequent provision of public information seems warranted from a social perspective, even if that may lead to an increase in volatility.

\section{Weak Complementarities}

Preferences and Technologies. The economy is populated by a continuum of measure one of agents, indexed by $i$ and uniformly distributed over the $[0,1]$ interval. Agents are risk neutral with utility

$$
u_{i}=A k_{i}-\frac{1}{2} k_{i}^{2}
$$

We interpret $k_{i} \in \mathbb{R}$ as individual investment (or effort), $A$ as the return to investment, and $k_{i}^{2} / 2$ as the cost of investment. We let $K=\int_{0}^{1} k_{i} d i$ denote the aggregate level of investment.

Like in Bryant (1983), Cooper and John (1988), Acemoglu (1993), Benhabib and Farmer (1994), and others, we introduce a complementarity by assuming that the individual return to investment is increasing in the aggregate level of investment:

$$
A=(1-\alpha) \theta+\alpha K
$$

The random variable $\theta$ parametrizes the exogenous return to investment (the underlying fundamentals of the economy) and the coefficient $\alpha \geq 0$ captures the degree of complementarity.

Finally, social welfare is given by a utilitarian aggregator, $w=\int_{0}^{1} u_{i} d i$. Using (1) and (2), we have that

$$
w=A K-\frac{1}{2} \int_{0}^{1} k_{i}^{2} d i=(1-\alpha) \theta K-(1-2 \alpha) \frac{1}{2} K^{2}-\frac{1}{2} v a r
$$

where $\operatorname{var}=\int_{0}^{1}\left(k_{i}-K\right)^{2} d i$ measures the cross-sectional heterogeneity in investment. Note that $w$ is concave in $K$ for $\alpha<1 / 2$, whereas it is convex for $\alpha>1 / 2$. In the latter case, lotteries would be welfare increasing and volatility in $K$ would be desirable. Since we are interested in the canonical

\footnotetext{
${ }^{2}$ Canzoneri (1985), Cukierman and Meltzer (1986), Atkeson and Kehoe (2001), Stokey (2002) and others consider how the transparency of policy instruments relates to the ability of the market to detect policy deviations in BarroGordon environments where the government lacks commitment. Our approach is clearly orthogonal to that line of research.
} 
case where welfare is decreasing in both volatility and heterogeneity, we restrict $\alpha \in[0,1 / 2)$. This also suffices for the equilibrium to be unique. ${ }^{3}$

Information and Transparency. The fundamentals $\theta \in \mathbb{R}$ are not known at the time investment decisions are made. Furthermore, agents have heterogenous beliefs about $\theta$. For simplicity, we let the common prior about $\theta$ be uniform over $\mathbb{R}$. We summarize the public information by a sufficient statistic $z$ such that $z=\theta+\sigma_{z} \varepsilon$, where $\varepsilon$ is standard normal, independent of $\theta$ and common across agents. Similarly, the private information of agent $i$ is summarized by a sufficient statistic $x_{i}=\theta+\sigma_{x} \xi_{i}$, where $\xi_{i}$ is standard normal, independent of $\theta$ and i.i.d. across agents. $\sigma_{z}$ and $\sigma_{x}$ parametrize the precision of public and private information, respectively.

Letting $\delta \equiv \sigma_{z}^{-2} /\left(\sigma_{x}^{-2}+\sigma_{z}^{-2}\right)$ and $\sigma \equiv\left(\sigma_{x}^{-2}+\sigma_{z}^{-2}\right)^{-1 / 2}$, the posterior belief of agent $i$ about $\theta$ is normal with mean $\mathbb{E}_{i}[\theta] \equiv \mathbb{E}\left[\theta \mid x_{i}, z\right]=(1-\delta) x_{i}+\delta z$ and variance $\operatorname{Var}_{i}[\theta] \equiv \operatorname{Var}\left[\theta \mid x_{i}, z\right]=\sigma^{2}$. Literally interpreted, the dependence of $\mathbb{E}_{i}[\theta]$ on $x_{i}$ is the result of the observation of private signals about $\theta$. More generally, however, $x_{i}$ introduces idiosyncratic variation in market expectations about the fundamentals and may thus be read also as heterogeneity in the filtering and interpretation of commonly available information. In this sense, $\delta$ measures the level of conformity in market expectations, whereas $\sigma$ the quality of available information.

In the following, we interpret an increase in the transparency of public information either as a reduction in $\sigma_{z}$ for given $\sigma_{x}$ (that is, an increase in the absolute precision of public information), or an increase in $\delta$ for given $\sigma$ (an increase in the relative precision of public information ${ }^{4}$ ). What we have in mind is that the transparency of public announcements, policy measures, and news in the media may affect either the noise in publicly available information, or the extent to which such information is interpreted differently across market participants. As it will become clear, our results are not very sensitive to which of the two interpretations we adopt.

\subsection{Equilibrium}

Each agent chooses $k_{i}$ so as to maximize $\mathbb{E}_{i}\left[u_{i}\right]$. It follows that the optimal investment is given by

$$
k_{i}=\mathbb{E}_{i}[A]=(1-\alpha) \mathbb{E}_{i}[\theta]+\alpha \mathbb{E}_{i}[K]
$$

Individual investment is thus increasing in the expected level of the fundamentals and in the expected level of aggregate investment.

\footnotetext{
${ }^{3}$ The equilibrium is unique if and only if $\alpha<1$. The case $\alpha \in[1 / 2,1)$ is considered in Angeletos and Pavan (2003). In that case, introducing noise in public information can be desirable to the extent that this substitutes for the absence of socially valuable lotteries.

${ }^{4}$ Note that $\delta$ is an increasing transformation of the relative precision of public information.
} 
Given the linearity of (3) and the normality of posterior beliefs about $\theta$, equilibrium investment decisions are linear so that $k_{i}=\beta x_{i}+\gamma z$, where $\beta$ and $\gamma$ are constants determined in equilibrium. Then, $K=\beta \theta+\gamma z$ and thus

$$
k_{i}=\mathbb{E}_{i}[A]=(1-\alpha+\alpha \beta)\left[(1-\delta) x_{i}+\delta z\right]+\alpha \gamma z
$$

It follows that $\beta=(1-\alpha)(1-\delta) /[1-\alpha(1-\delta)]$ and $\gamma=\delta /[1-\alpha(1-\delta)]$. Clearly, this is the unique symmetric linear (rational expectations) equilibrium. Furthermore, as proved in Morris and Shin (2002), when best responses are linear in $\mathbb{E}_{i}[\theta]$ and $\mathbb{E}_{i}[K]$, there do not exist equilibria other than this one. ${ }^{5}$ Hence,

Proposition 1 The equilibrium exists, is unique, and is given by $k_{i}=\beta x_{i}+\gamma z$, where

$$
\beta=1-\delta-\rho, \quad \gamma=\delta+\rho, \quad \text { and } \quad \rho=\frac{\alpha \delta(1-\delta)}{1-\alpha(1-\delta)} .
$$

Note that $\beta=1-\delta$ and $\gamma=\delta$ for $\alpha=0$. The term $\rho$ thus measures the excess sensitivity of equilibrium allocations to public information as compared to the case where there are no complementarities. Moreover, $\rho$ is increasing in $\alpha$. Stronger complementarities thus lead to a higher sensitivity of investment to public information. This is a direct implication of the fact that, in equilibrium, the public signal is a relatively better predictor of aggregate behavior than the private signal.

The equilibrium levels of volatility and heterogeneity are $\operatorname{Var}(K \mid \theta)=\left(\gamma \sigma_{z}\right)^{2}$ and $\operatorname{Var}\left(k_{i} \mid \theta, z\right)=$ $\left(\beta \sigma_{x}\right)^{2}$, respectively, where $\beta$ and $\gamma$ are given by (4). It follows that

Proposition 2 (i) Volatility necessarily increases with an increase in $\delta$ for given $\sigma$, and increases with a reduction in $\sigma_{z}$ for given $\sigma_{x}$ if and only if $\sigma_{z}^{2}>\frac{1}{1-\alpha} \sigma_{x}^{2}$. (ii) Heterogeneity falls with either an increase in $\delta$ or a reduction in $\sigma_{z}$.

This result may suggest that transparency can be socially undesirable when it increases volatility. However, we will see that welfare necessarily increases with an increase in either the relative or the absolute precision of public information.

\subsection{Welfare}

We now consider social welfare evaluated at equilibrium. This is given by $w(\theta)=\frac{1}{2} \theta^{2}-\frac{1}{2} \Omega$, where

$$
\Omega=\left(\beta \sigma_{x}\right)^{2}+(1-2 \alpha)\left(\gamma \sigma_{z}\right)^{2}=\operatorname{Var}\left(k_{i} \mid \theta, z\right)+(1-2 \alpha) \operatorname{Var}(K \mid \theta) .
$$

\footnotetext{
${ }^{5}$ Note that, although the two models are different, the structure of the best responses is identical and so are the equilibrium strategies.
} 
$\Omega$ measures the welfare consequences of heterogeneity in individual investment and volatility in aggregate investment. Since $\alpha<1 / 2$, welfare decreases with both heterogeneity and volatility. Furthermore, the relative weight on heterogeneity is increasing in $\alpha$. This reflects the social value of coordination: The stronger the complementarity, the more important the alignment in individual investment decisions.

Using (4) and substituting $\sigma_{x}=\sigma / \sqrt{1-\delta}$ and $\sigma_{z}=\sigma / \sqrt{\delta}$, we get

$$
\Omega=\frac{(1-2 \alpha)+\alpha^{2}(1-\delta)}{[1-\alpha(1-\delta)]^{2}} \sigma^{2} .
$$

It follows that $\alpha \in[0,1 / 2)$ suffices for $\Omega>0$ and therefore

Proposition 3 Welfare necessarily increases with either an increase in $\delta$ or a reduction in $\sigma$.

That welfare decreases with $\sigma$ for given $\delta$ follows directly from the fact that an increase in $\sigma$ means an increase in both volatility and heterogeneity. On the other hand, as shown in Proposition 2, an increase in $\delta$ implies lower heterogeneity at the expense of higher volatility. To understand why the effect of lower heterogeneity dominates, note that social welfare under a utilitarian objective coincides with the expected utility of an agent. Suppose for a moment that there were no complementarity $(\alpha=0)$, in which case $\beta=1-\delta$ and $\gamma=\delta$. It follows that $\Omega=\left(\beta \sigma_{x}\right)^{2}+\left(\gamma \sigma_{z}\right)^{2}=(1-\delta) \sigma^{2}+\delta \sigma^{2}=\sigma^{2}$, so that the expected utility of an agent depends only on $\sigma$ and not on $\delta$. This result should be expected: An increase in $\delta$ given $\sigma$ substitutes a higher precision in public information for a lower precision in private information, without altering the overall precision of information. When $\alpha=0$, individual choices are not interdependent and the decomposition of information between private and public is irrelevant. When instead $\alpha>0$, public information becomes more important than private information in predicting the return to investment, so that a substitution from private to public information raises the expected utility of an agent. In other words, an increase in $\delta$ raises welfare because it permits the agents to second guess each others' actions better and therefore facilitates more coordination in the market.

We next consider the comparative statics of $w$ with respect to $\sigma_{x}$ and $\sigma_{z}$, which combine the effects of $\delta$ and $\sigma$. To this aim, we first rewrite $\Omega$ as

$$
\Omega=\frac{\sigma_{x}^{2} \sigma_{z}^{2}\left[(1-2 \alpha) \sigma_{x}^{2}+(1-\alpha)^{2} \sigma_{z}^{2}\right]}{\left[\sigma_{x}^{2}+(1-\alpha) \sigma_{z}^{2}\right]^{2}} .
$$

It follows that

Proposition 4 (i) A reduction in $\sigma_{z}$ necessarily increases welfare. (ii) A reduction in $\sigma_{x}$ decreases welfare if and only if $\alpha>\frac{1}{3}$ and $\sigma_{x}^{2}>\frac{(1-\alpha)^{2}}{3 \alpha-1} \sigma_{z}^{2}$. 
Hence, more precise public information necessarily increases welfare. This is because a reduction in $\sigma_{z}$ for given $\sigma_{x}$ implies both better coordination (higher $\delta$ ) and lower uncertainty (lower $\sigma)$. On the contrary, more precise private information has an ambiguous effect on welfare. This is because a reduction in $\sigma_{x}$ for given $\sigma_{z}$ means lower uncertainty (lower $\sigma$ ) at the expense of lower coordination (lower $\delta$ ). If the complementarity is sufficiently weak, so that there is little value to coordination, or if the relative precision of public information is sufficiently low, so that volatility is low relative to heterogeneity, the benefit of lower uncertainty outweighs the cost of lower coordination. Otherwise, a reduction in $\sigma_{x}$ reduces welfare.

One can interpret $\sigma_{x}$ as the amount of information collected privately by market participants. Given the precision of the information and the strategies of the other agents, an individual decision maker always values more precise information. Hence, a lower $\sigma_{x}$ is always beneficial from a private perspective. On the other hand, an agent may prefer other agents to have less precise private information, since this would permit him to predict more accurately the aggregate level of investment. In other words, the private collection of information may create a negative externality, implying that a lower $\sigma_{x}$ need not be beneficial from a social viewpoint. We can thus have a market failure in the amount of private information collected by individual agents, in which case government intervention that discourages the collection of private information may actually increase welfare.

Finally, our result that more transparency in public information increases welfare even though it may also increase volatility, contrasts with the result of Morris and Shin (2002). As we anticipated in the introduction, the difference is due to the social value of coordination. In our model, the complementarity is equally present at the private and the social level. ${ }^{6}$ In Morris and Shin (2002), instead, there is an additional externality that perfectly offsets the complementarity at the social level. To see this, let $L=(\theta-A) K$ and suppose individual payoffs is $u_{i}=A k_{i}-\frac{1}{2} k_{i}^{2}+L$, in which case welfare reduces to $w=\int u_{i} d i=\int\left(\theta k_{i}-\frac{1}{2} k_{i}^{2}\right) d i$. This is the analogue of Morris and Shin (2002) in our setting. $L$ is the externality that renders the social (gross) return to investment independent of $K$, thus removing the complementarity at the social level. It follows that the coordination motive is not warranted from a social perspective, in which case stronger complementarity or more transparent public information may decrease welfare by exacerbating this motive. If instead coordination is socially valuable, as it is probably the case in economies with production or demand spillovers, network externalities, or incomplete financial markets, then more transparency is most likely to boost welfare.

\footnotetext{
${ }^{6}$ See also Angeletos and Pavan (2003) for further discussion.
} 


\section{Strong Complementarities}

In this section, we consider environments in which the complementarity is sufficiently strong to induce multiple equilibria for some information structures. To capture this possibility, we now let

$$
A=\theta+\mathbf{1}[K \geq r]
$$

where $\mathbf{1}[K \geq r]$ equals one if $K \geq r$ and zero otherwise. $r \in(0,1)$ represents the critical size of aggregate investment necessary for the complementarity to pay off; without serious loss of generality, we let $r=1 / 2$. For tractability, we also let the cost of investment be linear, so that

$$
u_{i}=A k_{i}-k_{i}
$$

and constrain $k_{i} \in[0,1]$.

Let $\underline{\theta} \equiv 0$ and $\bar{\theta} \equiv 1$. If $\theta \in[\underline{\theta}, \bar{\theta}]$ were common knowledge, both $k_{i}=1$ and $k_{i}=0$ for all $i$ would be an equilibrium; the former coincides with the first best, whereas the latter represents a coordination failure. With heterogeneous information, the possibility of multiple equilibria depends on the transparency of public information, as we show next.

\subsection{Equilibrium}

An agent finds it optimal to invest $k_{i}=1$ if $\mathbb{E}_{i}[A] \geq 1$, and $k_{i}=0$ otherwise. We restrict attention to equilibria with monotonic strategies, in which case for every $z$ there exists $x^{*}(z)$ such that $k_{i}=1$ if $x_{i}>x^{*}(z)$ and $k_{i}=0$ otherwise. Aggregate investment is then given by $K(\theta, z)=\Phi\left(\left[\theta-x^{*}(z)\right] / \sigma_{x}\right)$ and is thus increasing in $\theta$. This implies that $K \geq r(=1 / 2)$ if and only if $\theta \geq \theta^{*}(z)$, where $\theta^{*}(z)=x^{*}(z)$, and therefore $\mathbb{E}\left[A \mid x_{i}, z\right]=\mathbb{E}\left[\theta \mid x_{i}, z\right]+\operatorname{Pr}\left(\theta \geq \theta^{*} \mid x_{i}, z\right)$, or equivalently

$$
\mathbb{E}\left[A \mid x_{i}, z\right]=(1-\delta) x_{i}+\delta z+\Phi\left(\frac{(1-\delta) x_{i}+\delta z-\theta^{*}}{\sigma}\right) .
$$

It follows that $k_{i}=1$ if $x \geq x^{*}$, and $k_{i}=0$ otherwise, where $x^{*}$ solves $\mathbb{E}\left[A \mid x^{*}, z\right]=1$. Combining this with $\theta^{*}=x^{*}$, we conclude that the equilibrium threshold $x^{*}$ must solve

$$
F\left(x^{*} ; z, \delta, \sigma\right) \equiv \delta\left(z-x^{*}\right)-\sigma \Phi^{-1}\left[1-(1-\delta) x^{*}-\delta z\right]=0 .
$$

It is easy to check that the above always admits a solution. Let $\widehat{\delta} \equiv \sqrt{2 \pi} \sigma /(1+\sqrt{2 \pi} \sigma)$ and let $\widehat{\sigma}_{z}$ be the unique positive solution to $\sigma_{z}^{2} \sqrt{2 \pi}=\sigma_{x}^{2} \sqrt{\sigma_{x}^{-2}+\sigma_{z}^{-2}}$. If $\delta<\widehat{\delta}$, or equivalently $\sigma_{z}>\widehat{\sigma}_{z}$, then $F$ is monotonic in $x$ for all $z$, and thus the equilibrium threshold $x^{*}$ is unique. $F$ is instead non-monotonic whenever $\delta>\widehat{\delta}$, or equivalently $\sigma_{z}<\widehat{\sigma}_{z}$. In this case, let $\bar{z}, \underline{z}=$ 
$1 / 2 \pm\left\{m-\Phi^{-1}(m) \sigma(1-\delta) / \delta-1 / 2\right\}$, where $m>1 / 2$ solves $\phi\left(\Phi^{-1}(m)\right)=\sigma(1-\delta) / \delta$, and note that $0<\underline{z}<1 / 2<\bar{z}<1$. For $z \notin(\underline{z}, \bar{z})$, (5) has a unique solution $x^{*}$. But for $z \in(\underline{z}, \bar{z})$, (5) admits three solutions, $x_{\text {low }}^{*}<x_{\text {medium }}^{*}<x_{\text {high }}^{*}$. The two extreme solutions represent stable equilibria; the intermediate represents an unstable equilibrium, which we disregard. We conclude:

Proposition 5 (i) If $\delta<\widehat{\delta}$ (equivalently, $\sigma_{z}>\widehat{\sigma}_{z}$ ), or $z \notin(\underline{z}, \bar{z})$, there exists a unique threshold equilibrium: $k_{i}=1$ if and only if $x_{i} \geq x^{*}(z)$, where $x^{*}(z)$ is the unique solution to $F\left(x^{*} ; z, \delta, \sigma\right)=0$. (ii) If instead $\delta>\widehat{\delta}$ (equivalently, $\sigma_{z}<\widehat{\sigma}_{z}$ ) and $z \in(\underline{z}, \bar{z})$, there exist exactly two stable threshold equilibria: In the first, $k_{i}=1$ if and only if $x_{i} \geq x_{\text {low }}^{*}(z)$; in the second, $k_{i}=1$ if and only if $x_{i} \geq x_{\text {high }}^{*}(z)$, where $x_{\text {low }}^{*}(z)$ and $x_{\text {high }}^{*}(z)$ are the lowest and highest solutions to $F\left(x^{*} ; z, \delta, \sigma\right)=0$.

A high level of transparency (high $\delta$ or low $\sigma_{z}$ ) may thus lead to multiple equilibria. When this is the case, the second equilibrium $\left(x_{h i g h}^{*}\right)$ is characterized by less aggregate investment than the first $\left(x_{\text {low }}^{*}\right)$.

\subsection{Welfare}

The probability a coordination failure is possible depends on the transparency of information. Indeed,

$$
\operatorname{Pr}[z \in(\underline{z}, \bar{z}) \mid \theta]=\Phi\left(\frac{\bar{z}-\theta}{\sigma_{z}}\right)-\Phi\left(\frac{\underline{z}-\theta}{\sigma_{z}}\right)
$$

increases with $\bar{z}$, decreases with $\underline{z}$, and decreases with $\sigma_{z}$ for $\theta \in(\underline{z}, \bar{z})$. Moreover, $d \underline{z} / d \delta<0<$ $d \bar{z} / d \delta$. Hence,

Proposition 6 If $\delta>\widehat{\delta}$ (equivalently, $\sigma_{z}<\widehat{\sigma}_{z}$ ) and $\theta \in(\underline{z}, \bar{z}$ ), the probability of multiple equilibrium levels of investment increases with either a higher $\delta$ for given $\sigma$, or a lower $\sigma_{z}$ for given $\sigma_{x}$

An increase in the level of transparency may thus decrease welfare by increasing the probability that the "bad" equilibrium is played. Unfortunately, it is impossible to characterize welfare analytically. We thus resort to numerical simulations.

The effect of $\sigma_{z}$ on $w(\theta)$ is illustrated in Figure 1 for various values for $\theta$. The solid lines represent welfare along the high-investment equilibrium, whereas the dashed lines represent welfare along the low-investment equilibrium. In general, the welfare effects of transparency are neither monotonic nor homogeneous across $\theta$. However, the effects tend to be small for sufficiently low or sufficiently high values of $\theta$. We thus choose to concentrate on intermediate fundamentals. 
Since $\sigma_{z}=0$ restores common knowledge for any given $\sigma_{x}>0$, and since the "good" equilibrium under common knowledge coincides with the first best, it is trivial that welfare is maximized at $\sigma_{z}=0$. Therefore, provided that there is little risk of a coordination failure, full transparency is desirable. If instead the "bad" equilibrium is played whenever multiple equilibria are possible, welfare tends to decrease with a reduction in $\sigma_{z}$ beyond $\widehat{\sigma}_{z}$, in which case welfare is maximized at an intermediate level of transparency.

The patterns revealed by the examples of Figure 1 appear to be robust across a large number of simulations. Also, simulations of the welfare effect of $\delta$ give similar results and are omitted due to space limitations. We thus conclude

Proposition 7 If the socially preferable equilibrium is selected with high probability whenever multiple equilibria are possible, welfare is robustly maximal at $\delta=1$ for given $\sigma$, and necessarily maximal at $\sigma_{z}=0$ for given $\sigma_{x}$. If instead the worse equilibrium is selected with high probability, welfare is robustly maximized at $\delta \leq \widehat{\delta}$, or $\sigma_{z} \geq \widehat{\sigma}_{z}$.

\section{Concluding Remarks}

This paper examined the welfare effects of public and private information in an economy with investment complementarities. If the complementarity is weak so that multiple equilibria are never possible, more transparency in public information increases welfare, despite the fact that it also increases volatility. This is because more transparency facilitates more effective coordination in the market, which is socially valuable given that the complementarity is present at the aggregate level. On the other hand, if the complementarity is strong so that multiple equilibria are possible for high levels of transparency, more precise public information facilitates more effective market coordination on either equilibrium. In that case, "constructive ambiguity" becomes optimal when there is a high risk that the undesirable equilibrium is selected.

In Angeletos and Pavan (2003), we examine in more detail the properties of equilibrium and optimal allocations for economies with investment complementarities. We expect our insights to turn useful also in the analysis of other settings in which aggregate complementarities play an important role, such as economies with incomplete financial markets, Keynesian frictions, or network externalities. 


\section{References}

[1] Acemoglu, Daron (1993), "Learning About Others' Actions and the Investment Accelerator," Economic Journal 103, 318-328.

[2] Angeletos, George-Marios, Christian Hellwig, and Alessandro Pavan (2003), "Coordination and Policy Traps," MIT/UCLA/Northwestern mimeo.

[3] Angeletos, George-Marios, and Alessandro Pavan (2003), "The Value of Information and Coordination in Economies with Investment Complementarities," MIT/Northwestern mimeo.

[4] Atkeson, Andrew, and Patrick Kehoe (2001), "The Advantage of Transparent Instruments of Monetary Policy," NBER working paper.

[5] Benhabib, Jess, and Roger Farmer (1994), "Indeterminacy and Increasing Returns," Journal of Economic Theory 63, 19-41.

[6] Bryant, John (1983), "A Simple Rational Expectations Keynes-Type Model," Quarterly Journal of Economics 98, 3, 525-28.

[7] Canzoneri, Matthew (1985), "Monetary Policy Games and the Role of Private Information," American Economic Review 75, 1056-70.

[8] Cooper, Russell, and Andrew John (1988), "Coordinating Coordination Failures in Keynesian Models," Quarterly Journal of Economics 103, 441-463.

[9] Cukierman, Alex, and Allan Meltzer (1986), "A Theory of Ambiguity, Credibility, and Inflation under Discretion and Asymmetric Information," Econometrica 54, 1099-128.

[10] Hellwig, Christian (2003), "On the Aggregate Effects of Information Heterogeneity," UCLA mimeo.

[11] Lorenzoni, Guido (2003), "Imperfect Information and Aggregate Demand," Princeton University mimeo.

[12] Morris, Stephen, and Hyun Song Shin (2002), "The Social Value of Public Information", American Economic Review 92, 1521-1534.

[13] Stokey, Nancy (2002), "Rules versus Discretion After Twenty-Five Years," NBER Macroeconomics Annual 17, 9-45. 
[14] Woodford, Michael (2003), "Imperfect Common Knowledge and the Effects of Monetary Policy," in Knowledge, Information and Expectations in Modern Macroeconomics: in Honor of Edmund S. Phelps (P. Aghion, R. Frydman, J. Stiglitz, and M. Woodford, eds.). 

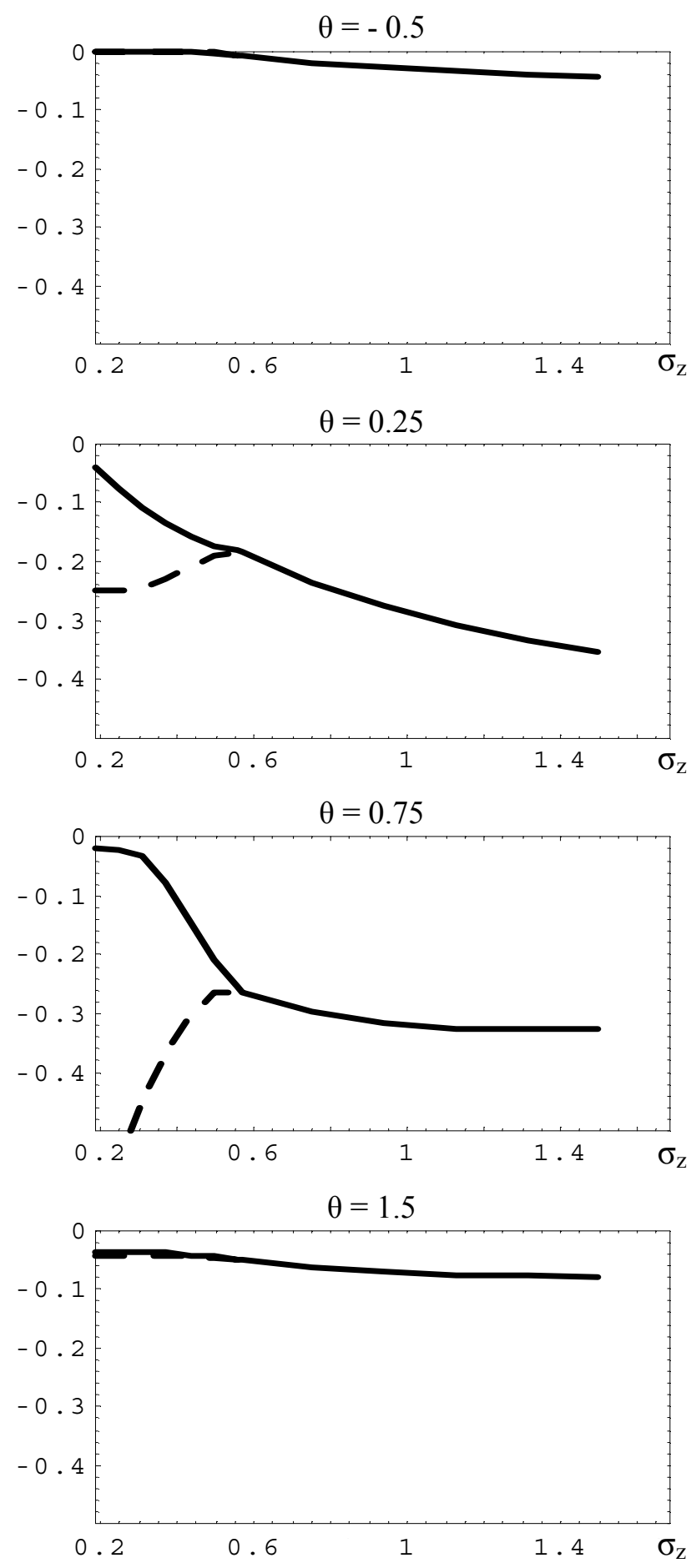

Figure 1

The effect of $\sigma_{z}$ on welfare. 